\title{
Best of intent, worst of both worlds: why sequentially combining epidemiological designs does not improve signal detection in vaccine safety surveillance
}

\author{
Faaizah Arshad ${ }^{1,2}$, Martijn J. Schuemie ${ }^{1,2.3}$, Evan P. Minty ${ }^{4}$, Thamir M. Alshammari ${ }^{5}$, Lana Y.H. \\ Lai $^{6}$, Talita Duarte-Salles ${ }^{7}$, Stephen Fortin ${ }^{3}$, Fredrik Nyberg ${ }^{8}$, Patrick B. Ryan ${ }^{2,3}$, George \\ Hripcsak $^{2,9,10}$, Daniel Prieto-Alhambra ${ }^{11,12}$, Marc A. Suchard ${ }^{1,2,13,14 *}$ \\ 1. Department of Biostatistics, University of California, Los Angeles, Los Angeles, CA, USA \\ 2. Observational Health Data Sciences and Informatics, New York, NY, USA \\ 3. Observational Health Data Analytics, Janssen R\&D, Titusville, NJ, USA \\ 4. O’Brien Institute for Public Health, Faculty of Medicine, University of Calgary, Calgary, \\ Alberta, Canada \\ 5. Medication Safety Research Chair, King Saud University, Riyadh, Saudi Arabia \\ 6. Division of Informatics, Imaging \& Data Sciences, University of Manchester, Manchester, \\ UK \\ 7. Fundació Institut Universitari per a la recerca a l'Atenció Primària de Salut Jordi Gol i \\ Gurina (IDIAPJGol), Barcelona, Spain \\ 8. School of Public Health and Community Medicine, Institute of Medicine, Sahlgrenska \\ Academy, University of Gothenburg, Gothenburg, Sweden \\ 9. Department of Biomedical Informatics, Columbia University, NY, NY, USA \\ 10. Medical Informatics Services, NewYork-Presbyterian Hospital, NY, NY, USA \\ 11. Centre for Statistics in Medicine, NDORMS, University of Oxford, Oxford, UK \\ 12. Health Data Sciences, Medical Informatics, Erasmus Medical Center University, \\ Rotterdam, NL \\ 13. Department of Human Genetics, University of California, Los Angeles, Los Angeles, CA, \\ USA \\ 14. VA Informatics and Computing Infrastructure, US Department of Veterans Affairs, Salt \\ Lake City, UT, USA

\section{* Correspondence:} \\ Marc A. Suchard \\ msuchard@ucla.edu
}

Keywords: vaccine surveillance, sensitivity, specificity, serial approach, safety signals 


\begin{abstract}
Background

Vaccine safety surveillance commonly includes a serial testing approach with a sensitive method for "signal generation" and specific method for "signal validation." Whether serially combining epidemiological designs improves both sensitivity and specificity is unknown.
\end{abstract}

\title{
Methods
}

We assessed the overall performance of serial testing using three administrative claims and one electronic health record database. We compared Type I and II errors before and after empirical calibration for historical comparator, SCCS, and the serial combination of those designs against six vaccine exposure groups with 93 negative control and 279 imputed positive control outcomes.

\section{Results}

Historical comparator mostly had lower Type II error than SCCS. SCCS had lower Type I error than the historical comparator. Before empirical calibration, serial combination increased specificity and decreased sensitivity. Type II errors mostly exceeded $50 \%$. After empirical calibration, Type I errors returned to nominal; sensitivity was lowest when the methods were combined.

\section{Conclusion}

We recommend against the serial approach in vaccine safety surveillance. While serial combination produced fewer false positive signals compared to the most specific method, it generated more false negative signals compared to the most sensitive method. Using the noisy historical comparator in front of SCCS deteriorated overall performance in evaluating safety signals.

\section{Key Messages:}

- Using the serial approach in vaccine safety surveillance did not improve overall performance: specificity increased but sensitivity decreased.

- Without empirical calibration, Type II errors exceeded 50\%; after empirical calibration, Type I error rates returned to nominal with negligible change to Type II error rates.

- While prior research has suggested high sensitivity of the historical comparator method in distinguishing true safety signals, there were cases when self-controlled case series was more sensitive.

- Vaccine safety surveillance is becoming increasingly important, so monitoring systems should closely consider the utility and sequence of epidemiological designs.

\section{$1 \quad$ Introduction}


medRxiv preprint doi: https://doi.org/10.1101/2022.02.18.22271183; this version posted February 22, 2022. The copyright holder for this preprint (which was not certified by peer review) is the author/funder, who has granted medRxiv a license to display the preprint in perpetuity. It is made available under a CC-BY 4.0 International license .

As of November 2021, over 7 billion doses of coronavirus disease 2019 (COVID-19) vaccines have been administered worldwide [1] [2], with 550 million in the United States (US) [3] [4] and 600 million in Europe [5]. While the rapid, global increase in vaccine uptake brings promise that the pandemic will end soon, undesirable and sometimes life-threatening adverse events can occur after immunization. Public health experts are employing post-vaccine safety surveillance to identify risk factors and complications of vaccine use.

Typically, in vaccine development, companies in the US are only permitted to market their vaccine if approved by the US Food and Drug Administration (FDA). However, due to the COVID19 emergency, the US FDA granted emergency use authorizations to permit vaccine distribution ahead of the typical approval process. This underscores the need for post-vaccine monitoring to ensure that unapproved vaccines in widespread use are safe.

Additionally, only a limited number of people participate in clinical trials (which are often highly restricted, such as with pregnant women or persons with specific comorbidities like cancer, etc.), so initial data on rare adverse events can be limited and ungeneralizable to real-world populations [6]. After the vaccine is marketed and provided to larger and diverse populations, as with the COVID-19 vaccine, previously undetected signals (possible associations between vaccines and adverse events that warrant further investigation) may become apparent [7].

The detection of such signals has traditionally been done via passive surveillance (spontaneous reporting systems) because they are somewhat inexpensive [8]. Some downsides, however, are they require health care practitioners to submit reports and are limited by underreporting or selective reporting [8]. A recently emerging alternative for generating vaccine safety evidence is through the use of electronic health records (EHRs), claims, and other available data. This approach has been divided into signal generation and signal evaluation [9], which are conducted using epidemiological designs such as historical comparator and self-controlled case series (SCCS). Whereas signal generation uses a highly sensitive but not specific method that can quickly generate hypotheses about signals, signal evaluation involves a complex and computationally expensive method that is highly specific but not sensitive. Monitoring programs that use a two-stage approach include the Vaccine Safety Datalink and the FDA Center for Medicare \& Medicaid Services (CMS). It is becoming difficult to distinguish between these two methods (one simple and one complex) as new softwares and analytic techniques are rapidly developed to investigate vaccine safety signals.

In this study, we evaluated whether the serial strategy (first applying a sensitive method and then a specific method) is useful for signal detection. Epidemiologists commonly use this method for exposure and outcome classification [10][11]. A "gold standard" serial testing method would have $100 \%$ sensitivity and specificity [12]. Prior studies have analyzed the statistical accuracy of combining diagnostic tests [13][14]; however, they are focused on understanding tests for patient-level screening, rather than population-level signals for surveillance, which are of interest here. Understanding signal detection for surveillance is relatively uncommon and new. 
It remains unknown whether and how using a serial strategy in vaccine surveillance based on available data improves the overall sensitivity and specificity of signal detection and validation, and therefore generates fewer false positive and false negative population-level signals. Specifically, we evaluated performance metrics when the historical comparator design (shown elsewhere to be relatively sensitive) [15] was followed by SCCS (shown elsewhere to be relatively specific) [16], using negative and imputed positive control outcomes in patients receiving historic vaccines across four national administrative claims and EHR databases [17].

\section{Materials and Methods}

\subsection{Databases}

To evaluate the comparative performance of epidemiological designs, we obtained deidentified clinical records from four large administrative claims and EHR databases that used the Observational Medical Outcomes Partnership (OMOP) common data model (CDM) in the United States: IBM MarketScan Commercial Claims and Encounters (CCAE), IBM MarketScan Medicare Supplemental Database (MDCR), IBM MarketScan Multi-State Medicaid Database (MDCD), and Optum ${ }^{\circledR}$ de-identified Electronic Health Record dataset (Optum EHR).

Of the four sources, three (IBM CCAE, IBM MDCR, and IBM MDCD) included adjudicated health insurance claims for commercially insured individuals younger than 65 years old, commercially insured individuals 65 years or older, and racially diverse Medicaid enrollees, respectively. Optum EHR contained electronic health records, covering the general US population. Please refer to Table 1 for additional details.

[Table 1 about here.]

\subsection{Exposures of interest}

We selected six historic vaccine exposures of interest: hemagglutinin type 1 / neuraminidase type 1 (2009 pandemic influenza) (H1N1pdm), seasonal influenza (Fluvirin), seasonal influenza (Fluzone), seasonal influenza (All), zoster (Shingrix; first or second dose), and human papillomavirus (HPV) (Gardasil 9; first or second dose). Each exposure was evaluated over a specific, historical, one-year start and end date (see Table 2).

[Table 2 about here.]

\subsection{Negative control outcomes}

We generated a single list of 93 negative control outcomes (see Supplementary Table 1), which were outcomes not believed to be caused by any vaccines of interest. To identify negative control outcomes that match the severity and prevalence of suspected vaccine adverse effects, we generated a candidate list of negative controls based on similarity of prevalence and percent of diagnoses that were recorded in an inpatient setting (as a proxy for severity). 
medRxiv preprint doi: https://doi.org/10.1101/2022.02.18.22271183; this version posted February 22, 2022. The copyright holder for this preprint (which was not certified by peer review) is the author/funder, who has granted medRxiv a license to display the preprint in perpetuity.

It is made available under a CC-BY 4.0 International license .

Clinicians reviewed the list to confirm their negative status. Effect size estimates for negative control outcomes should be close to the null; a detected signal away from the null would indicate Type I error.

\subsection{Positive control outcomes}

We modified the 93 negative controls to generate 279 imputed positive control outcomes (outcomes known to be caused by the vaccines) by multiplying the estimated effect size of each negative control by $1.5,2$, and 4 . A uniform effect size was used to compute estimates before either of the designs (historical comparator or SCCS) were applied. We chose imputed positive controls instead of real positive controls because well-established vaccine adverse events are rare or carefully monitored such that real-world data do not clearly convey the risk and magnitude of their associations. We used the imputed positive controls to evaluate Type II errors.

\subsection{Choice of epidemiological designs}

To assess the hypothesis that serial testing is favorable, we chose one epidemiological design suspected to be highly sensitive but not highly specific (historical comparator) [16]. Historical comparator compares the observed incidence of adverse events following immunization (AEFI) with the expected incidence of AEFI, estimated from an unexposed patient population, often before or "historical" to vaccine introduction. We adjusted for age and sex, as recommended by a previous study [18], and used a time-at-risk of 1-28 days after the historic visit (defined as a random outpatient visit) for both first and second doses. Any outcomes during that time were attributed to the vaccine [18]. Early signal detection during the historical comparator method can be done if some adverse events are present; however, this can also introduce confounding or false/missed signaling if background rates are inexact or change over time [19]. Then, we selected one design that was suspected to be specific but not sensitive (SCCS) [16]. SCCS compares the time shortly following vaccination to all other time in the same patient's record, therefore focusing our study on immediate or short-term adverse events [20] [21]. We used a SCCS design adjusted for age and season and excluded a 30-day pre-vaccination window from the analyses to account for healthy vaccinee bias [22].

\subsection{Performance metrics}

We computed effect size estimates with 95\% confidence intervals ( $\mathrm{Cls}$ ) and one-sided $\mathrm{P}$ values across all databases and all exposures for the historical comparator method alone, SCCS method alone, and the methods combined (historical comparator followed by SCCS). We distinguished signals from non-signals by using $P<0.05$ when both designs were applied separately, as well as when they were applied serially (with SCCS applied to signals generated by the historical comparator). 
medRxiv preprint doi: https://doi.org/10.1101/2022.02.18.22271183; this version posted February 22, 2022. The copyright holder for this preprint (which was not certified by peer review) is the author/funder, who has granted medRxiv a license to display the preprint in perpetuity. It is made available under a CC-BY 4.0 International license .

A primary concern in observational studies is the presence of systematic error that may exist because exposed and unexposed populations are not experimentally randomized, but simply observed [23]. Unlike random error, systematic error does not approach zero by merely increasing sample size [24]. It is therefore relatively more problematic when using large databases, such as in our study that captures records for millions of patients. Empirical calibration is a statistical procedure used to adjust for such systematic error [25]. It derives a null distribution from a sample of negative control outcomes and then applies the distribution to unknown effect size estimates, calibrating $P$ values so that $5 \%$ of negative controls have $P<$ 0.05 [24]. We can similarly calibrate $95 \%$ Cls by modifying negative controls to synthesize positive controls. After calibration, the coverage for a $95 \% \mathrm{Cl}$ is closer to the expected $95 \%$ [24]. Without calibration, certain biases may be left unaccounted for [25]. One important assumption of empirical calibration is that the systematic error of the exposure-outcome pair of interest draws from the same distribution as the systematic error for the negative control. Even though it does not require any negative control to have the exact same confounding structure as the exposure-outcome of interest, a weaker assumption of exchangeability is still required. Here we use P-value calibration and make no assumption about systematic error when the true effect size differs from the null.

We analyzed Type I and II errors before and after empirical calibration. A tradeoff exists between the two errors, leading to an increase in Type II error depending on how much systematic error is adjusted for.

Please refer here to access the protocol and analytic code.

\section{$3 \quad$ Results}

\subsection{Type I and II errors before empirical calibration for all databases}

Figure 1 reports Type I and II error rates in classifying positive and negative control outcomes across vaccine exposure groups. We further compare historical comparator and SCCS with the serial combination of those designs on the $y$-axis. Bars to the left of 0 indicate Type I error; bars to the right of 0 indicate Type II error. Type I error rate has a nominal cutoff of 0.05 as shown by the dotted line to the left of 0 . Error bars closer to 0 indicate higher sensitivity and specificity.

[Figure 1 about here.]

Almost all analyses had Type II error rates exceeding 50\% (Figure 1). Meanwhile, Type I error bars tended to range between $0 \%$ and $50 \%$, except for seasonal influenza (All) in the Optum EHR database where the historical comparator method generated many false positive signals.

The difference between Type I and II error rates before empirical calibration was particularly visible for the Optum EHR database. In most scenarios, the historical comparator method had higher sensitivity than SCCS or combined. When the historical comparator design was used to identify adverse events following vaccination, it generated fewer false negative signals (was less 
likely to miss a signal when it existed). Meanwhile, SCCS was more specific than the historical comparator method for H1N1pdm, seasonal influenza (All), seasonal influenza (Fluvirin), and zoster (Shingrix). The serial approach generated more false negative signals, while often reducing the number of false positives. This was consistent across all four databases: serial combination did not improve overall performance.

\subsection{Type I and II errors after empirical calibration for all databases}

We also considered how empirical calibration changed Type I and II errors (Figure 2). After calibration, Type I errors returned to nominal, and Type II errors increased in most cases. For the Optum EHR database, calibration visibly reduced Type I error rates of the historical comparator method for seasonal influenza (All). In spite of this, the combined method did not improve overall performance in the way that our hypothesis predicted. Among all three design choices and databases, sensitivity was lowest when the methods were serially combined (it increased the number of times that the surveillance system missed a signal when it actually existed). Yet, this may be expected since only signals flagged in the historical comparator method are then evaluated in SCCS, so there is an opportunity to decrease false positive signals.

[Figure 2 about here.]

\section{Discussion}

\subsection{Key results}

In this large-scale observational study of vaccine surveillance using four real-world databases in the US, we found advantages and disadvantages to serially combining sensitive and specific methods. We reject our hypothesis that combining designs increases sensitivity and specificity. Serial combination was helpful because it showed reduced Type I error, but also flawed because Type II error increased to be higher than when either of the methods were used alone. This tradeoff in Type I and II error is expected. Only signals flagged in the historical comparator were evaluated in the second method SCCS, creating an opportunity to remove false positive signals but not false negative signals. Also, SCCS typically already had quite favorable Type I error rates when applied individually, so combining methods did little to further decrease Type I error. Because our results showed false negative signals were excessively higher than false positive signals, the notion of dividing surveillance into two distinct stages of signal generation and evaluation, might not be ideal. We did not calculate minimum detectable relative risk (MDRR).

Although preferences for sensitivity and specificity trade-off may vary, an increase in specificity demonstrably did not offset a decrease in sensitivity. If specificity is high, but sensitivity is low, potential adverse events that might be caused by a vaccine can go overlooked, allowing for further circulation of the vaccine in the market. If sensitivity is high and specificity is low, 
medRxiv preprint doi: https://doi.org/10.1101/2022.02.18.22271183; this version posted February 22, 2022. The copyright holder for this preprint (which was not certified by peer review) is the author/funder, who has granted medRxiv a license to display the preprint in perpetuity. It is made available under a CC-BY 4.0 International license .

adverse events might be perceived as associated with a vaccine when in truth they are not, leading to the withdrawal of a vaccine from the market. In either case, an error has been made. Sensitivity alone does not predict enough about negative signals, and specificity alone does not predict enough about positive signals ("worst of both worlds").

Other studies have also examined performance characteristics in serial monitoring. Nelson et al. (2012) studied the use of serial approaches with observational EHR data in identifying signals between the DTap-IPV-Hib vaccine and adverse events [26]. One analytic challenge was confounding introduced from differences in vaccine uptake by age group [26]. Since observational studies are inherently uncontrolled, we corrected for confounding via empirical calibration.

Prior research has suggested that the historical comparator method is highly sensitive. For example, Li et al. recently found that historical comparisons had Type II errors ranging between $0 \%$ to $10 \%$ and Type I errors above 30\% [18]. Age and sex adjustment reduced Type I error, had little impact on Type II error, and improved precision in some cases [16]. Historical rate comparison was, overall, good at identifying true safety signals [16]. Based on Li et al.'s results, we evaluated the age and sex adjusted variant for historical comparator, using a time-at-risk of 1-28 days after the historic visit, for both first and second doses. However, contrary to this belief, we observed some instances where SCCS had lower Type II error and was therefore more sensitive than the historical comparator method. For example, in Figure 1, this was present for outcomes involving HPV (Gardasil 9) and zoster (Shingrix) in the Optum EHR and CCAE databases. There was also almost no distinguishable difference between the Type II errors for the historical comparator and SCCS methods in several of the plots, including for H1N1pdm and seasonal influenza (Fluvirin) in IBM MDCD, as well as seasonal influenza (Fluzone) in IBM MDCR. Likewise, there were some incidences where SCCS was less specific than historical comparator, such as in data for seasonal influenza (Fluzone) in Optum EHR, zoster (Shingrix) in IBM MDCR, and HPV (Gardasil 9) in CCAE. Comparisons of performance metrics demonstrate that the historical comparator is not always most sensitive. This uncertainty can make it difficult to predict the utility of a serial approach. Finally, Maro et al. (2014) conducted a similar study on sequential database surveillance using first an unmatched historical comparison method and second a self-controlled risk interval design (SCRI) [27]. Their time-at-risk was 1-42 days. However, this study implemented both methods using a sequential framework instead of serial approach and provided limited information on the sensitivity and specificity of combining methods.

On the individual patient-level scale, serial approach is common worldwide in the realm of diagnostic testing. Consider, for instance, detecting human immunodeficiency virus (HIV) prevalence in a population. A study amongst Nigerian women found that serial rapid tests (where the first HIV test has a sensitivity close to $100 \%$, and the second test is implemented only if the initial results were positive) were more sensitive and specific than conventional HIVtesting techniques [28]. Similar studies that used HIV rapid serological testing using data from Cote d'Ivoire [29], Uganda [30], and India [31] found that serial testing provides reliable results and has benefits in resource-limited areas. Serial testing has been widely adopted in clinical 
medRxiv preprint doi: https://doi.org/10.1101/2022.02.18.22271183; this version posted February 22, 2022. The copyright holder for this preprint (which was not certified by peer review) is the author/funder, who has granted medRxiv a license to display the preprint in perpetuity.

It is made available under a CC-BY 4.0 International license .

screenings for tuberculosis [32], prostate cancer [33], acute coronary syndrome [34], ovarian cancer [35], and recently COVID-19 [36]. This principle of investigating diseases using a cheap method before an expensive one is central to clinical medicine.

The importance of our study for the future of safety surveillance is related to recommended measures that should be addressed before using observational electronic healthcare databases for safety surveillance [37], namely that of pre-specifying and evaluating statistical designs. An accurate epidemiological design should overall generate the fewest number of false positive and false negative signals. Combining designs is unlikely to achieve this goal. Individuals who prioritize specificity over sensitivity might claim serial combination did work. Further studies should examine when this tradeoff is worthwhile. Since we observed variability in results, there should be similar analyses with other databases and exposures, such as COVID-19 vaccines or populations for which a certain occurrence has been defined (e.g. pregnant women or those with common comorbidities).

\subsection{Strengths and limitations}

Three strengths of this work are the use of real-world data, mapping of data to the OMOP CDM, and open-source availability of our code. First, real-world data represents a more heterogeneous population than randomized controlled trials that are tightly controlled. This improves the external validity of our data to the real-world patient population. Second, the CDM unifies data from multiple partners into a standardized format and enables us to pool analysis results from sources with large numbers of study subjects, without having to expose patient-level information. Third, the open-source availability of our code and protocol enables transparency and collaboration.

Our study is limited by the vaccine exposure of interest. While we seek to understand the use of epidemiological designs not only in general but also for COVID-19 vaccine surveillance, none of our exposures were COVID-19 vaccines. We addressed this limitation by choosing historical viral vaccines, which may be most similar. Another limitation is the use of imputed positive controls that assumes that systematic error does not change as a function of true effect size.

While Vaccine Safety Datalink and FDA CMS use serial combination with the best of intent, it may be the worst of both worlds (sensitivity and specificity). As COVID-19 vaccine safety surveillance becomes increasingly important, efforts to implement monitoring systems should carefully consider the utility and sequence of epidemiological designs.

\section{$5 \quad$ Data Availability Statement}

The protocol and analytical code underlying this manuscript are available in the Eumaeus Repository at https://github.com/ohdsi-studies/Eumaeus. 


\section{Ethics Statement}

This study did not require Institutional Review Board approval from data partners because it used de-identified patient data and did not constitute research with human subjects.

\section{$7 \quad$ Author Contributions}

FA, MJS, PBR, GH, DPA and MAS conceived the research. FA, MJS and MAS drafted the manuscript in consultation with EPM, TMA, LYHL, TDS, SF, FN, PBR, GH, DPA who provided critical feedback on the research and writing. All authors read and approved the final version.

\section{$8 \quad$ Funding}

This work was supported by US National Institutes of Health [grant number R01Al153044], a contract from the US Food \& Drug Administration and an Intergovernmental Personnel Act agreement with the US Department of Veterans Affairs.

\section{Conflict of Interest}

All authors have submitted the International Committee of Medical Journal Editors disclosure form. FA declares no competing interests. MAS receives grant funding from the US National Institutes of Health and the US Food \& Drug Administration and contracts from the US Department of Veterans Affairs and Janssen Research and Development. PBR, SF and MJS are employees of Janssen Research and Development and shareholders in Johnson \& Johnson. GH receives grant funding from the US National Institutes of Health and the US Food \& Drug Administration. DPA's research group has received grant support from Amgen, Chesi-Taylor, Novartis, and UCB Biopharma. His department has received advisory or consultancy fees from Amgen, Astellas, AstraZeneca, Johnson, and Johnson, and UCB Biopharma and fees for speaker services from Amgen and UCB Biopharma. Janssen, on behalf of IMI-funded EHDEN and EMIF consortiums, and Synapse Management Partners have supported training programmes organised by DPA's department and open for external participants organized by his department outside submitted work. FN was an employee of AstraZeneca until 2019 and owns some AstraZeneca shares. EPM, LYHL, TMA, and TDS declare no competing interests.

\section{Acknowledgments}

We are indebted to the entire EUMAEUS task force within the OHDSI community.

\section{References}


1. Bloomberg [Internet]. [cited 2021 Nov 18]. Available from: https://www.bloomberg.com/graphics/covid-vaccine-tracker-global-distribution/

2. Website [Internet]. Available from: Holder J. Tracking Coronavirus Vaccinations Around the World. The New York Times [Internet]. 2021 Jan 29 [cited 2021 Aug 16]; Available from: https://www.nytimes.com/interactive/2021/world/covid-vaccinations-tracker.html

3. CDC. COVID Data Tracker [Internet]. 2020 [cited 2021 Jul 28]. Available from: https://covid.cdc.gov/covid-data-tracker/

4. Holder J. Tracking Coronavirus Vaccinations Around the World. The New York Times [Internet]. 2021 Jan 29 [cited 2021 Aug 16]; Available from: https://www.nytimes.com/interactive/2021/world/covid-vaccinations-tracker.html

5. COVID-19 Vaccine Tracker [Internet]. [cited 2021 Jul 28]. Available from: https://vaccinetracker.ecdc.europa.eu/public/extensions/COVID-19/vaccine-tracker.html

6. Kim H-S, Lee S, Kim JH. Real-world Evidence versus Randomized Controlled Trial: Clinical Research Based on Electronic Medical Records. J Korean Med Sci. 2018 Aug 20;33(34):e213.

7. of Medical Sciences CFIO, of Medical Sciences CFIO, Others. Practical aspects of signal detection in pharmacovigilance: report of CIOMS Working Group VIII. Geneva: Council for International Organizations of Medical Sciences. 2010;

8. Kumar A, Khan H. Signal Detection and their Assessment in Pharmacovigilance. Open Pharm Sci J. 2015 Dec 17;2(1):66-73.

9. Mesfin YM, Cheng A, Lawrie J, Buttery J. Use of routinely collected electronic healthcare data for postlicensure vaccine safety signal detection: a systematic review. BMJ Glob Health. 2019 Jul 8;4(4):e001065.

10. [No title] [Internet]. [cited 2021 Nov 15]. Available from: https://cioms.ch/wpcontent/uploads/2018/03/WG8-Signal-Detection.pdf

11. R Silva I, Maro J, Kulldorff M. Exact sequential test for clinical trials and post-market drug and vaccine safety surveillance with Poisson and binary data. Stat Med. $2021 \mathrm{Sep}$ 30;40(22):4890-913.

12. Maxim LD, Niebo R, Utell MJ. Screening tests: a review with examples. Inhal Toxicol. 2014 Nov;26(13):811-28.

13. Pepe MS, Thompson ML. Combining diagnostic test results to increase accuracy. Biostatistics. 2000 Jun;1(2):123-40.

14. Thompson ML. Assessing the diagnostic accuracy of a sequence of tests. Biostatistics. 2003 Jul;4(3):341-51. 
15. Website [Internet]. Available from: Schuemie MJ, Arshad F, Pratt N, Nyberg F, Alshammari TM, Hripcsak G, et al. Vaccine safety surveillance using routinely collected healthcare data - An empirical evaluation of epidemiological designs [Internet]. bioRxiv. medRxiv; 2021. Available from: http://medrxiv.org/lookup/doi/10.1101/2021.08.09.21261780

16. Schuemie MJ, Arshad F, Pratt N, Nyberg F, Alshammari TM, Hripcsak G, et al. Vaccine safety surveillance using routinely collected healthcare data - An empirical evaluation of epidemiological designs [Internet]. bioRxiv. medRxiv; 2021. Available from: http://medrxiv.org/lookup/doi/10.1101/2021.08.09.21261780

17. Protocol.utf8 [Internet]. [cited 2021 Sep 30]. Available from: https://ohdsistudies.github.io/Eumaeus/Protocol.html

18. Li X, Lai LYH, Ostropolets A, Arshad F, Tan EH, Casajust P, et al. Bias, precision and timeliness of historical (background) rate comparison methods for vaccine safety monitoring: an empirical multi-database analysis [Internet]. bioRxiv. medRxiv; 2021. Available from: http://medrxiv.org/lookup/doi/10.1101/2021.07.10.21258463

19. Yih WK, Kulldorff M, Fireman BH, Shui IM, Lewis EM, Klein NP, et al. Active surveillance for adverse events: the experience of the Vaccine Safety Datalink project. Pediatrics. 2011 May;127 Suppl 1:S54-64.

20. Whitaker HJ, Paddy Farrington C, Spiessens B, Musonda P. Tutorial in biostatistics: the selfcontrolled case series method [Internet]. Vol. 25, Statistics in Medicine. 2006. p. 1768-97. Available from: http://dx.doi.org/10.1002/sim.2302

21. Petersen I, Douglas I, Whitaker H. Self controlled case series methods: an alternative to standard epidemiological study designs [Internet]. BMJ. 2016. p. i4515. Available from: http://dx.doi.org/10.1136/bmj.i4515

22. Weldeselassie YG, Whitaker HJ, Farrington CP. Use of the self-controlled case-series method in vaccine safety studies: review and recommendations for best practice. Epidemiol Infect. 2011 Dec;139(12):1805-17.

23. Schuemie MJ, Ryan PB, DuMouchel W, Suchard MA, Madigan D. Interpreting observational studies: why empirical calibration is needed to correct p-values. Stat Med. 2014 Jan 30;33(2):209-18.

24. Schuemie MJ, Hripcsak G, Ryan PB, Madigan D, Suchard MA. Empirical confidence interval calibration for population-level effect estimation studies in observational healthcare data. Proc Natl Acad Sci U S A. 2018 Mar 13;115(11):2571-7.

25. Schuemie MJ, Hripcsak G, Ryan PB, Madigan D, Suchard MA. Robust empirical calibration of p-values using observational data. Stat Med. 2016 Sep 30;35(22):3883-8.

26. Nelson JC, Yu O, Dominguez-Islas CP, Cook AJ, Peterson D, Greene SK, et al. Adapting group 
sequential methods to observational postlicensure vaccine safety surveillance: results of a pentavalent combination DTaP-IPV-Hib vaccine safety study. Am J Epidemiol. 2013 Jan $15 ; 177(2): 131-41$.

27. Maro JC, Brown JS, Dal Pan GJ, Kulldorff M. Minimizing signal detection time in postmarket sequential analysis: balancing positive predictive value and sensitivity. Pharmacoepidemiol Drug Saf. 2014 Aug;23(8):839-48.

28. Mbachu II, Udigwe G, Joseph I, John O, Samuel UO, Joseph U, et al. The evaluation of accuracy of serial rapid HIV test algorithm in the diagnosis of HIV antibodies among pregnant women in south east Nigeria. BMC Res Notes. 2015 Oct 12;8:557.

29. Koblavi-Dème S, Maurice C, Yavo D, Sibailly TS, N'guessan K, Kamelan-Tano Y, et al. Sensitivity and specificity of human immunodeficiency virus rapid serologic assays and testing algorithms in an antenatal clinic in Abidjan, Ivory Coast. J Clin Microbiol. 2001 May;39(5):1808-12.

30. Galiwango RM, Musoke R, Lubyayi L, Ssekubugu R, Kalibbala S, Ssekweyama V, et al. Evaluation of current rapid HIV test algorithms in Rakai, Uganda. J Virol Methods. 2013 Sep;192(1-2):25-7.

31. Iqbal HS, Solomon S, Murugavel KG, Solomon SS, Balakrishnan P. Evaluation of two indigenous rapid and two ELISA assays for the diagnosis of HIV infection India. Indian J Med Microbiol [Internet]. 2012 [cited 2021 Dec 17];30(4). Available from: https://pubmed.ncbi.nIm.nih.gov/23183462/

32. Moses MW, Zwerling A, Cattamanchi A, Denkinger CM, Banaei N, Kik SV, et al. Serial testing for latent tuberculosis using QuantiFERON-TB Gold In-Tube: A Markov model. Sci Rep. 2016 Jul 29;6(1):1-8.

33. Smith DS, Catalona WJ, Herschman JD. Longitudinal screening for prostate cancer with prostate-specific antigen. JAMA. 1996;276(16):1309-15.

34. Zhelev Z, Ohtake H, Iwata M, Terasawa T, Rogers M, Peters JL, et al. Diagnostic accuracy of contemporary and high-sensitivity cardiac troponin assays used in serial testing, versus single-sample testing as a comparator, to triage patients suspected of acute non-STsegment elevation myocardial infarction: a systematic review protocol. BMJ Open. 2019 Mar 30;9(3):e026012.

35. Berek JS, Bast RC Jr. Ovarian cancer screening. The use of serial complementary tumor markers to improve sensitivity and specificity for early detection. Cancer. 1995 Nov 15;76(10 Suppl):2092-6.

36. Kobayashi T, Trannel A, Holley SA, Alsuhaibani MA, Abosi OJ, Jenn KE, et al. Coronavirus Disease 2019 Serial Testing Among Hospitalized Patients in a Midwest Tertiary Medical Center, July-September 2020. Clin Infect Dis. 2020 Oct 29;73(9):e3116-9. 
medRxiv preprint doi: https://doi.org/10.1101/2022.02.18.22271183; this version posted February 22, 2022. The copyright holder for this preprint

(which was not certified by peer review) is the author/funder, who has granted medRxiv a license to display the preprint in perpetuity.

It is made available under a CC-BY 4.0 International license.

37. Nelson JC, Wellman R, Yu O, Cook AJ, Maro JC, Ouellet-Hellstrom R, et al. A Synthesis of Current Surveillance Planning Methods for the Sequential Monitoring of Drug and Vaccine Adverse Effects Using Electronic Health Care Data. EGEMS (Wash DC). 2016 Sep

6;4(1):1219.

\section{Figure Captions}

Figure 1. We depict uncalibrated Type I and II errors across all databases using all exposures and negative and positive controls during a time-at-risk of 1-28 days following vaccination. Generally, the historical comparator had low Type II error. SCCS had low Type I error. Clinical intuition would predict the combined method to be highly sensitive and specific, but results showed high specificity and low sensitivity.

Figure 2. We depict Type I and II errors after empirical calibration across all databases using all exposures and negative and positive controls during a time-at-risk of 1-28 days following vaccination. Type I errors returned to nominal. Type II errors mostly increased. Even with empirical calibration, the serial approach did not increase overall performance of a two-stage surveillance program. 
Tabla 1: Three administative alaime and one EHR data scurses with dotaile on population, patients, and hiatery

Data Source Population Patients History Description

IBM MarketScan Commercial Claims and Encounters Database (CCAE)
Commercially insured, $<65$ years

Enrollees in US employer-sponsored insurance health plans. Acjucicated health insurance claims ie.g- inpatient, outpatient, and outpetient phormacy), lab tests, and enrollment date from lerge employers and haalth plans who provide private healticare coverage. Includes varicus fee-for-service, preferred provider orgarizations, and capitated health plans.

\section{IBN Heaith} MarketScan" MuitState Medicaid Database (MDCD)

\section{Medicaid} enrollees, racially diverse
$142 \mathrm{M} 2000$

\section{IBM MarketScan}

Medicare Supplementsl and Coordination of Eonefits Database (MDCR)

\section{Commercially insured, 65+ 10M years}

Health services of retirees in US with primary or Medicare supp-lemental coverage through privately insured fee-for-service, point-of-service, or capitated health plans. Adjudicated health insurance claims (e.g. inpatient, put patient, and outpatient pharmacy). Lab tests for some.
Optum de-identified Electronic Health Record Dataset (Optum EHR)
Derived from dozens of healthcare provider organizations in US (700+ hospitals and $7,000+$ clinics treailing $103+$ million patients). US, general $\quad 93 \mathrm{M} \quad 2006-\quad$ Clinical information, prescriptions, lab results, vital signs, body measuremen1s, diagnoses, procedures, and information derived from clinical Notes using Natural Language Processing. 
Table 2: Six historic (group5 of) vaccines evaluated over specific one-year start and end dates

\section{Exposure Name \\ Shot Start Date \\ End Date \\ History Start Date History End Date}

H1N1 vaccination

First Sep 1, 2009 May 31, 2010

Sop 1, 2008

Sep 1, 2016

Sep 1, 2016

Sep 1, 2016

Jan 1,2017

HPY vaccination (Gardasil 9) Both Jan 1,2018 Dec 31, $2018 \quad$ Jan 1, 2017
Refer to Appendix A of the study protocol for the format cohort definitions of each exposure.

HPV vaccination (Gardasil 9) Both Jan 1,2018 Dec 31, 2018 Jan 1, 201
Refer to Apperdix A of the study protocol for the format cohort definitions of each exposure.

Both Jan 1, 2018 Dec 31, 2018

Jan 1.2017

\begin{tabular}{l} 
HPV vaccination (Gardasil 9) Both Jan 1, 2018 Dec 31, 2018 Jan 1, 20 \\
\hline Refer to Appendix A of the study protocol for the format cohort definitions of each exposure.
\end{tabular}
May 31,2009

May 31,2017

May 31, 2017

May 31,2017

Dec 31, 2017

Dec 31, 2017 